\title{
"It's Me as a Person, Not Me the Disease": Patient Perceptions of an HIV Care Model Designed to Engage Persons with Complex Needs
}

\author{
Kristin Beima-Sofie, PhD, MPH, ${ }^{1}$ Emily R. Begnel, MPH, ${ }^{1}$ Matthew R. Golden, MD, MPH ${ }^{2-4}$ \\ Allison Moore, $\mathrm{BS},{ }^{3}$ Meena Ramchandani, MD, ${ }^{2,3}$ and Julia C. Dombrowski, MD, $\mathrm{MPH}^{2-4}$
}

\begin{abstract}
Ending the HIV epidemic will require dedicated efforts to engage the highest need persons living with HIV (PLWH) in treatment. We assessed patient perceptions of a clinic in Seattle, Washington, that is designed for PLWH who do not engage in conventional HIV care. The Max Clinic provides walk-in access to care, incentives for blood draws and achieving viral suppression, and intensive case management. We conducted semistructured individual interviews with 25 patients purposively recruited to obtain diverse viewpoints. Interviews were audio-recorded and transcribed. Analysis used a constant comparative approach to identify major themes related to the components of the program. For many participants, engagement in the Max Clinic was the first time they had success with HIV treatment. Relationships with clinic staff and the ability to receive care on a walk-in basis had the strongest influences on engagement. Participants felt that Max Clinic staff attended to their social circumstances in ways that were distinct from prior care experiences. Walk-in visits removed perceived stigma associated with failure to keep appointments and provided immediate attention to acute concerns. Financial incentives initially motivated participants to attend clinic and take medications, but were less important for supporting ongoing engagement in care. Food incentives motivated patients to seek care and helped them focus on health issues. In summary, patients identified walk-in access to care, monetary and food incentives, and relationships with clinic staff, particularly case managers, as the key elements of an HIV clinic model for high-need PLWH.
\end{abstract}

Keywords: HIV care continuum, engagement in care, substance use, homelessness, mental health

\section{Introduction}

I NCOMPLETE ENGAGEMENT in HIV care and treatment among persons living with HIV (PLWH) is a central barrier that must be overcome to achieve the goals of the Ending the HIV Epidemic initiative in the United States. ${ }^{1}$ Although barriers to care that PLWH experience have been well described and synthesized in the literature, ${ }^{2-4}$ few interventions have been demonstrated to re-engage individuals who have fallen out of care. ${ }^{5}$ Most re-engagement interventions described to date have focused on changing patient care-seeking behavior or supporting patients in navigating the current HIV health care system. ${ }^{6-10}$ Less work has been done to change the health care environment to meet the needs of patients who are not well engaged in conventional care. ${ }^{11-13}$ PLWH may be less likely to stay in care if they encounter difficulty scheduling appointments, long wait times, and stigma and discrimination in the health care setting. ${ }^{14-16}$ Those who are homeless or unstably housed, have substance use disorders, or have psychiatric disorders are particularly vulnerable to disengaging from HIV care cr, $^{2,17,18}$ and may need options that differ from the conventional clinic approach. The differentiated care strategy responds to this need by adapting the treatment approach to meet the needs of the patient. ${ }^{12,13,19}$ With this approach, services are intensified for those who are unstable and at higher risk of morbidity and mortality.

The Max Clinic in Seattle, Washington, is a walk-in, incentivized care model that was designed to engage high-need

\footnotetext{
${ }^{1}$ Department of Global Health, University of Washington, Seattle, Washington, USA.

${ }^{2}$ Department of Medicine, University of Washington, Seattle, Washington, USA.

${ }^{3}$ HIV/STD Program, Public Health-Seattle \& King County, Seattle, Washington, USA.

${ }^{4}$ Department of Epidemiology, University of Washington, Seattle, Washington, USA.
} 
PLWH who are virally unsuppressed, have complex medical and social barriers to care, and do not successfully re-engage in HIV care with lower intensity outreach interventions. ${ }^{20,21}$ The clinic includes walk-in visits for primary care, financial (cash) incentives for completing visits with blood draws and for viral suppression, snacks, food vouchers, no-cost cell phones and bus passes, and intensive case management with cross-agency service coordination. ${ }^{20}$ The clinic includes both medical case managers (social workers) and nonmedical case managers (health department disease intervention specialists) whom patients can access by phone or walk-in visits 5 days per week. Walk-in medical visits are available five afternoons per week with one of a team of Infectious Disease physicians. Patients who enroll in the Max Clinic are more than three times as likely to achieve viral suppression in the year after enrollment compared with virally unsuppressed patients enrolled in conventional HIV care. ${ }^{21}$ However, because the Max Clinic was implemented as a combination intervention, the mechanism of effect is uncertain and it is not clear which elements are essential to this approach.

To gain insight into how and why the walk-in, incentivized care approach works, we conducted a qualitative study to elucidate patient perspectives on the Max Clinic and each component of the intervention. Understanding patient experiences in care and perspectives on the Max Clinic features can inform optimization of the intervention and implementation of similar approaches in other settings. While the interviews focused on patients' experiences with the Max Clinic, the themes are broadly relevant to efforts to engage high-need PLWH, and perhaps other high-need patients with chronic diseases, in medical care.

\section{Methods}

\section{Study design and population}

We conducted semistructured individual interviews (IDIs) from February to May 2017 with 25 PLWH enrolled in the Max Clinic. The design of the Max Clinic model is consistent with the Behavioral Model for Vulnerable Populations ${ }^{22}$ and the model of HIV health services utilization described by Ulett et al., ${ }^{23}$ updated by Hawk et al. ${ }^{11}$ The intervention addresses domains of the health care environment relevant for vulnerable populations, such as access/availability/convenience, administrative hassle, care coordination and continuity, as well as personal domains such as competing needs, hunger, living conditions, and transportation. We included cash incentives on a theoretical basis consistent with the principles of contingency management ${ }^{24}$ at a time when little research addressing the impact of incentives on HIV outcomes was available. ${ }^{25}$

\section{Recruitment}

All patients attending the Max Clinic for $\geq 6$ months were eligible for inclusion. We purposively recruited patients to represent a range of life experiences and clinical care outcomes, including gender, race/ethnicity, housing status, substance use, and viral suppression. We also sought to enroll a subset of participants who had not been fully engaged in the Max Clinic. Participants were informed of the study by case managers or medical providers, and those who affirmed interest were referred to the study coordinator, who was not associated with the Max Clinic apart from this project. Participants were primarily recruited as they showed up for clinic visits, but to meet prespecified targets among patient subgroups, some were recruited through phone calls inviting them to come in for the study.

This study was approved by the University of Washington Institutional Review Board. All participants provided written informed consent.

\section{Data collection}

Semistructured IDI guides containing open-ended questions were developed collaboratively between study team members, based on Max Clinic features, literature reviews, and experiences, providing HIV care to this population. IDI guides captured beliefs and experiences related to the main topic areas of (1) experiences receiving care at the Max Clinic, (2) experiences accessing HIV care and treatment before enrollment in the Max Clinic, (3) life experiences that might influence engagement in health care, and (4) suggestions for improving the Max Clinic. All interviews began with openended questions soliciting feedback on the Max Clinic generally, followed by directive questioning to elicit more specific information about each clinic feature or incentive. Interviews specifically asked patients to reflect on their interactions and relationships with the clinical care team at the Max Clinic and how these may have differed from relationships in other HIV clinics where they had previously received care. All interviews were audio-recorded and transcribed verbatim.

IDIs were conducted by a trained social worker who was not involved in providing services for any of the participants. Before IDIs, the facilitator was briefed on the dynamics of the patient population, clinic, and HIV epidemic in Seattle. The facilitator was instructed to remain neutral about the Max Clinic during the IDIs. The facilitator informed all study participants that identifiable information from the interviews would not be shared with staff from the clinic and that interview participation would not affect their clinical care.

A total of 25 IDIs were completed. IDIs were conducted in a quiet, private area of the Max Clinic and lasted an average of $39 \mathrm{~min}$. Prespecified and response-driven probes were used to provide the richest data possible. Data were collected using a modified version of the constant comparison approach, ${ }^{26}$ where the facilitator took detailed notes during the IDIs that were immediately shared with team members, allowing key themes and concepts from initial IDIs to inform data collected during future IDIs. All documents were reviewed by the data analysis team and brief summaries of key themes were generated for each IDI.

\section{Data analysis}

Analysis focused on identifying the key components of Max Clinic success. We analyzed data using a combination of conventional and directed content analysis ${ }^{27}$ to produce a description of key concepts and themes arising within and between individual participants, and within and between Max Clinic features. An initial codebook was developed deductively based on the specific features of the Max Clinic, and life and clinical care experiences probed in the semistructured IDI guides. Open coding was then used to generate an additional subset of codes. The codebook was refined through an iterative process of reviewing subsets of 
transcripts against codebook drafts and swapping transcripts between analysis team members. All transcripts were independently coded by one member of the analysis team using a final version of the codebook. Coded transcripts were all reviewed by another member of the analysis team for accuracy, and disagreements in code applications were resolved through group discussion until consensus on code application was reached. Each member of the analysis team reviewed and coded at least half of the transcripts. Dedoose was used for data management and analysis. After all data were coded, investigators ran queries to extract data on attributes affecting engagement in care (current and past) and components of the Max Clinic. Initial themes were situated within the updated HIV health services utilization model and used to characterize the most influential features of Max Clinic and how these features minimized barriers to engagement in care.

\section{Results}

Of the 25 participants, 14 identified as male, 8 as female, and 3 as transgender (Table 1). The median age was 37 (IQR: 34-50) years. Most participants identified themselves as either non-Hispanic black $(36 \%)$ or white $(52 \%)$. The majority (64\%) reported crystal methamphetamine (meth) use, either in combination with other drugs $(n=7)$ or alone $(n=9)$. Only five participants $(20 \%)$ reported no stimulant, opioid, or hazardous alcohol use. Most participants were on antiretroviral therapy $(80 \%)$ and had suppressed HIV viral loads $(60 \%)$ at the time of the interview.

All participants described positive experiences accessing and engaging in HIV care since enrolling in the Max Clinic.

Table 1. Characteristics of Qualitative InTERVIEW PARTICIPANTS ENROLLED IN THE MAX Clinic $(N=25)$

\begin{tabular}{lc}
\hline Characteristic & $\mathrm{n}(\%)$ or Median (IQR) \\
\hline Age (years) & $37(34-50)$ \\
Gender & $14(56 \%)$ \\
Male & $8(32 \%)$ \\
Female & $3(12 \%)$ \\
Transgender & \\
Race/ethnicity & $13(52 \%)$ \\
Non-Hispanic white & $9(36 \%)$ \\
Non-Hispanic black & $3(12 \%)$ \\
Other & \\
Substance use & $9(36 \%)$ \\
Methamphetamine & $7(28 \%)$ \\
Methamphetamine and opioids & $3(12 \%)$ \\
Opioids & $1(4 \%)$ \\
Hazardous alcohol use & $5(20 \%)$ \\
None & \\
Housing status & $15(60 \%)$ \\
Unhoused & $7(28 \%)$ \\
Housed-stable & $3(12 \%)$ \\
Housed-unstable & $273(119-368)$ \\
CD4 count (cells/mm $\left.{ }^{3}\right)$ & $15(60 \%)$ \\
Virally suppressed & $20(80 \%)$ \\
Taking antiretroviral & \\
medications & \\
\hline
\end{tabular}

${ }^{\mathrm{a}}$ HIV RNA level $<200$ copies/mL at time of last measurement. $\mathrm{IQR}$, interquartile range.
Several participants described care received from the Max Clinic as life-altering. For many, it was the first time they were successful with treatment, and they valued the impact treatment success had on their life. Others described feeling connected to the clinic staff, comparing the Max Clinic to a supportive, family-like environment. Participants described the Max Clinic as a place they felt valued, listened to, and treated as an equal.

\begin{abstract}
"I was there at that road where I felt like giving up every day, and I used to tell the doctors that too.....I was like, 'You don't understand how hard it is to take [antiretroviral medications]. You don't understand that my mom's dead and sometimes I don't care to even live'..... When I came here, it just changed my life. So, I am forever grateful to be here."-Participant 16

“They help me help myself. And even if I don't want to help myself, they're still in it. They're here for me regardless. And they make it known....they go to the max of when it comes to my care."-Participant 24
\end{abstract}

Three major themes emerged from the IDIs related to how the Max Clinic improved care engagement related to HIV health services utilization barriers: (1) walk-in visits lowered barriers to care access, (2) incentives encouraged patients to prioritize care and medication adherence, and (3) coordinated social services and support met patient needs in real time.

\section{Walk-in visits lowered the barrier to accessing care and alleviated shame associated with missing scheduled appointments}

One of the most valued features of the Max Clinic was the walk-in clinic appointments. Participants noted that their social and living situations, including homelessness, substance use, and lack of transportation, made it difficult for them to keep scheduled appointments. When trying to engage in traditional care, participants felt that their constantly shifting life situations made it too challenging to stick with appointments made weeks or months in advance.

\footnotetext{
"It's hard over there [at the other HIV clinic] because they'll make an appointment, like, two weeks out, rather than the Max Clinic, you come in anytime you want, and I like the drop-in, and you're guaranteed to see a doctor, which I love, you know, because my life has a time schedule thing." Participant 9
}

When they did show up for appointments in their prior HIV clinics, they often felt that clinic interactions were rushed and there was not enough time to spend with their providers. When they did not show up, they felt embarrassed that they had made a mistake, felt judged by the providers at their next appointment, and were then deterred from returning.

\footnotetext{
"When I don't have an appointment to miss, it doesn't put me off of coming back. Because I feel very flaky when I miss appointments. I feel like I'm not meeting my standards and I don't want to face the music with that and go back in. I will avoid stuff that's critical to my health because I'm embarrassed."-Participant 21
}

In contrast, patients liked how providers and case workers were always available at the Max Clinic, that the clinic space was a comfortable and safe space to stay, and that providers took time to listen to them during appointments. Patients also 
noted that walk-in appointments allowed them to get immediate attention for issues that were concerning them.

"I love that I don't have appointments. I just walk in, I come when I feel like I need them, or just to talk. And like I said, it's like family here, except better."-Participant 15

“It works out quite well. I don't have to worry, 'When is my next appointment? I forgot to ask this question last time I saw them and now I have to wait 3 months.' But instead, this way, I can remember and I can come back and say, 'Hey, this is what's going on.'......And that way, they can deal with the problem right then and there, instead of waiting for the problem to even get worse."-Participant 7

\section{Incentives encouraged patients to prioritize care and medication adherence}

Participants had complex life circumstances that made it difficult to prioritize medical care. The majority of participants described at least some time in their life during which substance use was problematic for them. While not necessarily motivated to change their substance use, many recognized that substance use negatively impacted their adherence to HIV medications and routine clinic attendance.

\begin{abstract}
"Relapsing on crystal meth doesn't really help me take my meds. It hurts my ability to remember. For a while, I was not taking my meds because I wanted to get really sick to kind of draw attention to that fact that the housing crisis in Seattle is pretty F-ed up. I mean, I don't really have a problem taking my meds when I'm not getting tweaked out on meth. Like, I'm pretty good."-Participant 21
\end{abstract}

Participants appreciated receiving financial incentives for blood draws and as a reward for viral suppression. Financial incentives helped participants reprioritize actions and behaviors and elevated the value participants placed on medical care seeking and medication adherence.

\begin{abstract}
"When they sent me here and they told me that [financial incentives] was one of the options if I get my blood drawn and I take my medicine more...... because I've always had problems taking my medication for my whole life...... light bulb just clicked in my head and was like, 'I need that money. You need to take your medicine.' So, that's what really helped me overcome the pills."-Participant 16
\end{abstract}

Despite the ability of financial incentives to encourage appropriate health seeking and adherence behaviors among participants, most participants placed lower value on financial incentives than on how they were treated by clinic staff. When asked to describe the most important features of the clinic, almost all participants described positive interactions with clinic staff. When specifically probed to describe their thoughts on financial incentives received, many participants noted that while they appreciated receiving the incentives, they viewed the incentives as an added bonus rather than as critical for ensuring treatment success.

\footnotetext{
"Well, it's not that important, but it's an incentive. Keeps me on my toes. Makes sure I take my meds, you know?"Participant 7
}

Some participants noted that financial incentives played an important role when the participants first came to the Max Clinic and motivated them to take medications, but the importance lessened over time.

\begin{abstract}
"It's a great incentive for you to take your meds and get in the habit of taking your meds, so you know, even when....you're at the point where you leave this [the Max Clinic] and you stop getting cash for taking them, you're already in the habit of [taking them] and are already in a routine of taking them, so it's not that big of a deal for you to continue to take them, you know?"-Participant 5
\end{abstract}

Other participants viewed the financial incentives as adding to the intrinsic satisfaction they received from achieving viral suppression.

On the financial incentive for viral suppression: "It was important because it made my spirits feel good.... and it was like I got a surprise for even helping myself, like I got a reward for doing something that I needed to do and once you get that, it makes you even feel better. Like, 'Oh, I did it!', and plus I get initiatives to do it, so it makes you keep on wanting to take your medicine and.....you got doctors that want you to keep taking your medicine and support where they say, 'Hey, I'll give you, you know, initiatives and then you get this.' It feels good!"-Participant 9

Participants also described being excited about how their success in HIV treatment translated to success in other life domains, including relationships with friends and family members.

\begin{abstract}
"It means a lot, and it means a lot to my mom, and for my mom to be proud of me, that says a lot, you know? And I've been waiting for her to say that she's proud of me for a long time.....And that's why I thank God for the Max Clinic, because I never thought I'd hear those words out of my mom's mouth. And my life isn't perfect to this day, but it's better than it was a year ago, mentally, emotionally, physically. I feel better, in every way."-Participant 11
\end{abstract}

Participants also received snacks, food vouchers, bus passes, and cell phones. Snacks provided in the clinic, such as chips and sports drinks, and \$10 meal vouchers after visits were important to many participants because they were homeless and experiencing food insecurity. Participants described often being hungry and without other options to get food. Participants noted that food helped them focus on health issues, rather than hunger, during the clinic visit.

\begin{abstract}
"I'm very hungry a lot of the time. I'm homeless. I live in a tent and I don't eat a lot sometimes and I'm very hungry always and to be able to come here and get some snacks is nice. It just even helps with being able to be interviewed by a doctor and have everything firing cognitively because your blood sugars are good. It's just important to eat and it's nice to be able to."-Patient 21
\end{abstract}

Even patients with stable housing and the ability to prepare food for themselves valued the opportunity to have someone cook for them and felt cared for by receiving a meal.

\footnotetext{
"I'm a good old Southern boy. I'm a good old cook, child. But every now and then, you want somebody else to feed you. And they do just fine over there at the cafeteria. I love it. On days....coming off from the weekend or whatever, I come here and get a voucher just so I don't have to cook that evening, you know, and five dollars can pretty much cover close to damn near a nice tall soda and a hamburger and fries. That's usually what I always order because their burgers over there are slamming."-Participant 23
}

Participants valued the bus pass because it allowed them to get to the clinic from anywhere in the city, whenever needed, 
without money. The bus passes were a highly valued commodity to homeless patients, allowing them to get to places in the city faster than walking, their usual mode of transportation.

\begin{abstract}
“'It's been real important. I wouldn't be able to here every day that I' $m$ here if I didn't have it, because I can't afford the bus every day. So, yeah, that's definitely awesome, you know?" Participant 5

"It helps a lot. Especially since I'm out further away from the hill [location of clinic] than I'm used to. I can manage before usually. I just talk to the bus drivers or, you know, give them some money. As much as I can. But this makes it a lot easier. And I don't have to worry about them kicking me off of the bus."-Participant 12
\end{abstract}

Not all participants we interviewed had received a cell phone through the clinic, but those who had placed lower value on cell phones than bus passes, primarily because the phones were often stolen or lost soon after being received.

"I did get a cell phone and it took me awhile to get used to it, because I'm not used to having a phone and I really didn't need one.... and I've lost it or someone stole it from me." Participant 12

The few participants who described using the cell phones found them helpful for staying in contact with family members and clinic providers.

"[The cell phone] was important for me because [the clinic] was able to get in touch with me for the Max Clinic purposes. So, like I said, I have temporary memory loss. So, I forget. So, that's wonderful for me, so they can be able to contact me. And if the phone rings, I know that it's them. I know that it's something that I-that it's important, so yeah, it was great to have the iPhone."-Participant 24

\section{Coordinated social services and support met patient needs in real time}

Homelessness or unstable housing, as well as problematic substance use, impacted social relationships and posed challenges to seeking HIV care for many participants. Case managers and doctors who were willing to listen and help sort out social situations had a significant impact on patient's experiences with HIV care.

\begin{abstract}
"I've put them through a trial, because I was a mess when I first got here, being homeless and all that. I remember I broke down one day. My birthday last year. I couldn't take it no more. They just helped get me through it. So, [the Max social worker] has been my sister ever since."-Participant 15

"Just the little things that they do, I do notice and it just makes an impact for how a person views the whole medical profession. You can tell that they are doing things because they want to do things to make a difference and they're there wholeheartedly."-Participant 17
\end{abstract}

Patients often felt like the case managers on the Max Clinic team were some of the only people to treat them with respect and view them not just as someone living with HIV.

"It's like, you go home and you think about them. I never went home and thought about my doctors before, you know? Just how nice and concerned [they are] about your health. Not just, HIV ....it's me as a person. Not me the disease. So, that helps a lot. And I would refer friends, but I don't have any that have the same problems that I do."-Participant 15

\begin{abstract}
"They wanted her [a case manager] to walk me over there to make sure I got my breathing treatment. So, she stayed and we was there for a long time too....She shared some information with me, so that was personal, and we talked about my situation and what I was going through. So, it was more [than just the accompaniment] when I was there, she was a real person that I can express my emotions to, what I'm going through, and she could give positive feedback to me. Because really, I don't talk to people about me being sick, so it felt good for me to even have that little time with her to actually be able to talk to somebody."-Participant 9
\end{abstract}

\section{Discussion}

This qualitative study of patients enrolled in a walk-in, incentivized HIV clinic for high-need PLWH found that walk-in visits lowered barriers to accessing HIV care, incentives helped patients to prioritize clinic visits and medication adherence, and relationships with case managers provided a crucial source of support in patients' lives beyond meeting medical needs. Participants described past experiences of difficulty completing prescheduled appointments and experiences of stigma for missing appointments that discouraged them from re-engaging in care. The "here and now" approach of the Max Clinic walk-in visits was a better fit with the reality of their lives. Patients expressed heterogeneous opinions about the importance of the incentives, but the meal vouchers were particularly appreciated, either as a means to temporarily relieve hunger from food insecurity or as a special treat to anticipate after clinic visits. Financial incentives initially motivated reprioritization of clinical care, but the importance wore off over time for many participants who experience personal satisfaction from achieving viral suppression. The personalized support that patients received was a crucial factor contributing to patients' experiences of dignity and being cared for as a whole person.

This study adds to the prior literature on important elements of a care model for patients with complex barriers to HIV medical care. Stigma and discrimination related to HIV have been well described as barriers to HIV care. ${ }^{16}$ However, the participants in our interviews described a different type of stigma they experienced in HIV clinics, before enrolling in the Max Clinic, that negatively impacted their engagement in HIV care: embarrassment and shame from missing scheduled appointments. This result is consistent with a report describing how the "good" patient ideal poses a barrier to engagement in HIV care for some vulnerable patients, ${ }^{28}$ and that patients sometimes perceive long clinic wait-times as a form of discrimination. ${ }^{2}$ This finding suggests that, at least for some patients, structural changes to health care delivery can alleviate the sense of shame associated with missing appointments and remove the perceived stigma against "bad" patients. Further, when patients arrive late for scheduled appointments, clinic visits are often rushed by necessity to keep to a schedule, but participants in our study described how being rushed through visits in prior HIV clinics left them feeling uncared for. A walk-in model of care is not only logistically easier for patients with unpredictable life circumstances, but, combined with skilled clinical staff, can also alleviate the shame some patients feel in conventional HIV clinics.

One theme that arises from these interviews is the concept of financial incentives as a bridge to engagement with a more 
comprehensive clinical intervention, such as the Max Clinic. Evidence for the impact of financial incentives on HIV care outcomes in the United States is mixed. For example, the Test, Link-to-Care Plus Treat study (HPTN 065) used financial incentives broadly applied to all patients enrolled in the intervention clinics and found a statistically significant, but relatively small, impact on viral suppression, which was greater in clinics with initially lower rates of viral suppression. ${ }^{25}$ In contrast, the Max Clinic is tailored for a small subset of PLWH who are not successfully engaged in conventional HIV care, and financial incentives targeted to highneed patients may have a stronger effect. Metsch et al. studied the impact of targeted financial incentives combined with peer navigation compared with usual care or peer navigation alone. ${ }^{6}$ While the financial incentives were ongoing at 6 months after enrollment, the combined impact of peer navigation and financial incentives on viral suppression was statistically significant compared with standard care, although the effect disappeared by 12 months, after the intervention was stopped. Together with existing literature, the Max Clinic experience suggests that financial incentives targeted to high-need patients can play a role in increasing HIV care engagement and viral suppression, and that the effect is likely to be greater and more sustainable if incentives are part of a more comprehensive intervention.

The importance participants placed on food vouchers, snacks, and bus passes provides support for the idea that incentives for care engagement do not need to be monetary. Instead, incentives that attend to basic unmet needs, including food insecurity and lack of transportation, can encourage clinical care seeking behaviors. By meeting the basic needs of patients, patients are able to reprioritize their energy to focus on clinical care, rather than finding food or transportation, therefore facilitating improved engagement.

Many patients view their relationships with the Max Clinic case managers and doctors as the most important part of the Max Clinic. Public health disease intervention specialists may not be the most appropriate staff for this type of work in all areas, but our findings suggest that it is crucial to have staff whose primary role is intensive, personalized support distinct from that of a medical case manager or social worker. In our study, participants valued the relationships with staff that were not entirely focused on their medical or social work needs, but the broader aspects of their lives, resulting in the sense of being treated as "me, the person, not me the disease."

Our study was conducted with a small group of patients in one clinic, which may limit its generalizability. However, even though we focused on the Max Clinic, participants expressed values about medical care that are broadly relevant for programs designed to reach patients with chronic diseases who have complex medical and social needs. Although we attempted to include a variety of patients who could provide diverse perspectives, we enrolled only a subset of patients and our findings may not represent the views of all patients enrolled in the Max Clinic. Nonetheless, this study is an important contribution to the literature because it included patients who have been poorly engaged in care or who failed to be engaged by prior outreach attempts. This group is a high-priority population in the context of efforts to end the HIV epidemic that is difficult to recruit and often underrepresented in research.
In summary, we found in this qualitative study of "hardto-reach" patients enrolled in a low-barrier HIV clinic previously demonstrated to increase viral suppression that patients' view of the essential elements of the care model includes: (1) walk-in visits to lower the barrier to access; (2) incentives to encourage patients to prioritize care visits and medication adherence, in part, by satisfying unmet basic needs that compete with the importance of HIV care; and (3) relationships with case managers and medical providers who attend to patients' complex psychosocial needs. Novel HIV clinic services and models of care delivery designed specifically to engage high-need complex patients are crucial for realizing the goals of the national Ending the HIV Epidemic initiative.

\section{Acknowledgments}

We are grateful to Megan Touhey, MSW, for conducting the qualitative interviews and reviewing transcripts of the interviews for accuracy. We would like to thank the patients of the Max Clinic who participated in the study and provided our study team with detailed stories of their lives. We would also like to thank the Max Clinic staff for their assistance with study recruitment and continued dedication to the provision of high-quality HIV care to PLWH with complex medical and social needs.

\section{Author Disclosure Statement}

J.C.D. has conducted research supported by grants to the University of Washington from Hologic.

\section{Funding Information}

The following grants supported this study: National Institutes of Health grants R03 DA 042668, P30AI027757, and P30 AI 027757-28S1.

\section{References}

1. Fauci AS, Redfield RR, Sigounas G, Weahkee MD, Biroir BP. Ending the HIV epidemic: A plan for the United States. JAMA 2019;321:844-845.

2. Hall BJ, Sou KL, Beanland R, et al. Barriers and facilitators to interventions improving retention in HIV care: A qualitative evidence meta-synthesis. AIDS Behav 2017;21: 1755-1767.

3. Bulsara SM, Wainberg ML, Newton-John TRO. Predictors of adult retention in HIV care: A systematic review. AIDS Behav 2018;22:752-764.

4. Bulsara SM, Wainberg ML, Audet CM, Toby RON-J. Retention in HIV care in Australia: The perspectives of clinicians and clients, and the impact of medical and psychosocial comorbidity. AIDS Patient Care STDs 2019;33: 415-424.

5. International Advisory Panel on Optimizing HIV Care Continuum Outcomes. IAPAC guidelines for optimizing the HIV care continuum for adults and adolescents. J Int Assoc Provid AIDS Care 2015;14 Suppl 1:S3-S34.

6. Metsch LR, Feaster DJ, Gooden L. Effect of patient navigation with or without financial incentives on viral suppression among hospitalized patients with HIV infection and substance use: A randomized clinical trial. JAMA 2016;316:156-170. 
7. Giordano TP, Cully J, Amico KR, et al. A randomized trial to test a peer mentor intervention to improve outcomes in persons hospitalized with HIV infection. Clin Infect Dis 2016;63:678-686.

8. Wohl AR, Ludwig-Barron N, Dierst-Davies R, et al. Project engage: Snowball sampling and direct recruitment to identify and link hard-to-reach HIV-infected persons who are out of care. J Acquir Immune Defic Syndr 2017;75: 190-197.

9. Irvine MK, Chamberlin SA, Robbins RS, et al. Come as you are: Improving care engagement and viral load suppression among HIV care coordination clients with lower mental health functioning, unstable housing, and hard drug use. AIDS Behav 2017;21:1572-1579.

10. Dombrowski JC, Hughes JP, Buskin SE, et al. A cluster randomized evaluation of a health department data to care intervention designed to increase engagement in HIV care and antiretroviral use. Sex Transm Dis 2018;45:361-367.

11. Hawk M, Coulter RWS, Egan JE, et al. Exploring the healthcare environment and associations with clinical outcomes of people living with HIV/AIDS. AIDS Patient Care STDS 2017;31:495-503.

12. Roy M, Bolton Moore C, Sikazwe I, Holmes CB. A review of differentiated service delivery for HIV treatment: Effectiveness, mechanisms, targeting, and scale. Curr HIV/AIDS Rep 2019;16:324-334.

13. Teasdale CA, Yuengling K, Preko P, et al. Persons living with HIV with advanced HIV disease: Need for novel care models. J Int AIDS Soc 2018;21:e25210.

14. Holtzman CW, Shea JA, Glanz K. Mapping patientidentified barriers and facilitators to retention in HIV care and antiretroviral therapy adherence to andersen's behavioral model. AIDS Care 2015;27:817-828.

15. Lam Y, Westergaard R, Kirk G, et al. Provider-level and other health systems factors influencing engagement in HIV care: A qualitative study of a vulnerable population. PLoS One 2016;11:e0158759.

16. Geter A, Herron AR, Sutton MY. HIV-related stigma by healthcare providers in the United States: A systematic review. AIDS Patient Care STDS 2018;10:418-424.

17. Aidala AA, Wilson MG, Shubert V, et al. Housing status, medical care, and health outcomes among people living with HIV/AIDS: A systematic review. Am J Public Health 2016;106:e1-e23.

18. Rooks-Peck CR, Adegbite AH, Wichser ME, et al. Mental health and retention in HIV care: A systematic review and meta-analysis. Health Psychol 2018;37:574-585.
19. World Health Organization. What's New in Service Delivery; Fact Sheet: HIV Treatment and Care. 2016, Geneva, Switzerland. Available at: https://www.who.int/hiv/pub/ arv/arv2015-service-delivery-factsheet/en/ (Last accessed November 25, 2019).

20. Dombrowski JC, Ramchandani M, Dhanireddy S, et al. The Max Clinic: Medical care designed to engage the hardestto-reach persons living with HIV in Seattle and King County, Washington. AIDS Patient Care STDS 2018;32: 149-156.

21. Dombrowski JC, Galagan S, Ramchandani R, et al. HIV care for patients with complex needs: A controlled evaluation of a walk-in, incentivized care model. Open Forum Infect Dis 6:ofz294.

22. Gelberg L, Andersen RM, Leake BD. The Behavioral Model for Vulnerable Populations: Application to medical care use and outcomes for homeless people. Health Serv Res 2000;34:1273-1302.

23. Ulett KB, Willig JH, Lin HY, et al. The therapeutic implications of timely linkage and early retention in HIV care. AIDS Patient Care STDS 2009;23:41-49.

24. Davis DR, Kurti AN, Skelly JM, et al. A review of the literature on contingency management in the treatment of substance use disorders, 2009-2014. Prev Med 2016;92:36-46.

25. El-Sadr WM, Donnell D, Beauchamp G, et al. Financial incentives for linkage to care and viral suppression among HIV-positive patients: A randomized clinical trial (HPTN 065). JAMA Intern Med 2017;177:1083-1092.

26. Glaser B, Strauss A. The Discovery of Grounded Theory, Strategies for Qualitative Research. Chicago, IL: Aldine Publishing Company, 1967.

27. Hsieh HF, Shannon SE. Three approaches to qualitative content analysis. Qual Health Res 2005;15:1277-1288.

28. Koester KA, Johnson MO, Wood T, et al. The influence of the 'good' patient ideal on engagement in HIV care. PLoS One 2019; 14:e0214636.

Address correspondence to: Julia C. Dombrowski, MD, MPH Department of Medicine University of Washington 325 Ninth Avenue, Box 359777 Seattle, WA 98104 USA

E-mail: jdombrow@uw.edu 
